\title{
Moving forward with motion reduction, detection and correction in cardiac PET
}

\author{
Ernest V. Garcia, PhD, ${ }^{a}$ and Jonathon A. Nye, $\mathrm{PhD}^{\mathrm{a}}$ \\ a Department of Radiology and Imaging Sciences, Emory University, Atlanta, GA
}

Received Mar 1, 2021; accepted Mar 1, 2021

doi:10.1007/s12350-021-02599-6

\section{See related article, pp. 1596-1606}

The heart is a moving organ, depending on heart rate, situated in the chest that is moving due to respiratory motion. The lungs during the heavier breathing (larger tidal volume) associated with stress tend to push the tip of the heart downwards which then slowly creeps upward in its return to rest. Patient's body tends to move during scanning, usually along the axis of the table, mostly due to physical and/or mental discomfort. The magnitudes of these movements can be significant and highly variable between patients. Our instincts might betray us to accept that motion is a natural component of the imaging process and thus that the best a diagnostician can do is "read around" the problems caused by these motions. As PET scanner technology continues to improve leading to improvements in image quality such as is the case with digital photon counting and time of flight reconstruction, our instincts might also mislead us into expecting a reduction in the detrimental effects of motion. These improvements in image quality actually make motion artifacts more apparent. ${ }^{1}$ Consider the analogy that you replace your old standard TV with an ultra-high definition set. With this ultra-high definition now you see more blemishes in the face of your favorite news anchor. The ultra-high definition image did not create the blemishes; it just made them easier to see. Understanding the effect that motion creates on our images is important both for improving visual interpretation but more significantly for developing motion

Reprint requests: Ernest V. Garcia, PhD, Department of Radiology and Imaging Sciences, Emory University, 101 Woodruff Circle, Room 1203, Atlanta, GA 30322; ernest.garcia@emory.edu

J Nucl Cardiol 2022;29:1607-10.

1071-3581/\$34.00

Copyright (C) 2021 American Society of Nuclear Cardiology. correction algorithms that can overcome these effects and improve quantification.

These motions create two related types of negative visual and quantitative effects on our images; image degradation and image artifacts. Image degradation is the loss in resolution and thus image quality due to the smearing that takes place when the shutter of a camera remains open while the subject is moving. In our case the PET scanner is the camera and the left ventricle is the subject. Image artifact is an artificial finding in an image that should not be there, often leading to an incorrect interpretation and thus an incorrect diagnosis. Three well-documented types of motion artifacts in PET that lead to image artifacts are PET-emission-CTtransmission misregistration, ${ }^{2-4}$ motion during dynamic acquisition $^{5-9}$ and motion due to breathing. ${ }^{10-12}$

Emission-transmission misregistration happens when using the CT transmission from a PET/CT scanner to correct for attenuation of the $511 \mathrm{keV}$ photons along the body during PET imaging. This misregistration is due to changes in the position of the heart in relation to the thorax between the PET acquisition and the CT acquisition, these two acquisitions being sequential rather than simultaneous and of different temporal sampling. Most PET/CT misregistration leads to falsepositive defects in the LV anterior and lateral wall and to a lesser degree in the apical and inferior wall depending on the location of the misregistration. ${ }^{2}$ Both manual and automatic algorithms ${ }^{3}$ have been developed to correctly re-register the PET/CT imagery resolving in most cases these artifacts. Importantly, joint reconstruction of emission PET image with simultaneous registration of the attenuation map from the CT transmission image using Time-of-Flight data is a promising new tool to provide perfectly aligned PET/CT imagery. ${ }^{4}$

More recently, motion effects have been reported in dynamic PET acquisitions used in myocardial blood flow quantification. ${ }^{5-9}$ Myocardial blood flow (MBF) and flow-reserve (MFR) measurements generally require a volume-of-interest to define the blood pool to measure 
the input function and myocardial volume-of-interest to measure the tissue concentration over time. Misalignments between the input function VOI and the blood pool during the first pass transit of the tracer have been shown to result in low mean global MBF and MFR errors but in very significant errors in mean MBF and MFR in the right coronary artery vascular territory when the misalignment was in the inferior LV direction. ${ }^{5}$ Flow errors due to misalignment between the myocardial VOI and the myocardial tissue during the late tissue phase seem to be small. ${ }^{5}$ Several investigators are actively developing and validating tools to detect and correct for the LV displacements between frames during dynamic PET acquisitions. ${ }^{5-9}$

Methods to correct for the motion effects that degrade image quality due to breathing are also being developed. ${ }^{10-12}$ A simple method is one that uses respiratory gating that tracks the respiratory cycle from inspiration to expiration. A more advanced method is one that uses external triggers to select a fraction of the perfusion data that is well registered with the attenuation image. ${ }^{1}$ Both these methods suffer from lower count statistics and thus noisy data. Improved methods for correcting for the breathing motion are designed to use all the counts in the entire cardiac PET acquisition. These are usually data-driven approaches ${ }^{10,11}$ that use the entire set of PET events to measure motion vectors that are used during reconstruction to generate motionfrozen images ${ }^{12}$ correcting for most effects including respiration.

Existing motion correction algorithms applied to dynamic cardiac PET acquisitions usually only assess frame-to-frame shifts, mostly neglecting intra-frame motion blurring, i.e. motion that takes place within each frame. It is this intra-frame motion blurring that degrades the quality of our static cardiac PET images used for detecting hypoperfusion. In this issue of the Journal Armstrong et al report on the motion correction performance of their version of a data-driven motion correction algorithm designed to correct intra-frame motion blurring developed as part of research collaboration with industry. ${ }^{13}$ This novel data-driven motion correction algorithm, named DDMC, is based on extracting information from list-mode PET to track heart motion in mm with $1 \mathrm{~s}$ time resolution and apply the detected displacement vectors along the table direction to correct motion within a static perfusion images and/or an individual frame within a dynamic acquisition. Their previous work ${ }^{14}$ presented phantom validation and preliminary clinical feasibility data for the DDMC method, and the present article reports on their evaluation of the impact of motion blurring of varying degrees in a consecutive series of 300 patients in order to determine the prevalence of image degradation due to motion in rest and stress [Rb-82]-Chloride cardiac PET studies, including the prevalence of non-diagnostic images due to excessive motion.

These investigators' assessments were done by visual analysis between the DDMC motion corrected reconstructions and those without motion correction. They found motion degradation in $58 \%$ of the stress static images and $33 \%$ of the rest images with a $2 \%$ rate of non-diagnostic studies due to motion. Remarkably, their DDMC algorithm was successful in identifying and tracking the heart in all images and thus presumably correcting and/or improving all images leading the

Table 1. Tools for motion reduction, detection and correction in cardiac PET

\begin{tabular}{|c|c|c|c|}
\hline Tool & Concern & Type of Motion & Refere \\
\hline Pre-scan patient information & Mental discomfort & Patient motion & \\
\hline $\begin{array}{l}\text { Patient comfortably } \\
\text { positioned }\end{array}$ & Physical discomfort & Patient motion & \\
\hline Regadenoson stress & $\begin{array}{l}\text { Heart displacement with } \\
\text { adenosine }\end{array}$ & Cardiac creep & 15 \\
\hline $\begin{array}{l}\text { Manual \& Automatic PET/CT } \\
\text { registration }\end{array}$ & $\begin{array}{l}\text { Emission/transmission } \\
\text { misregistration }\end{array}$ & $\begin{array}{l}\text { LV position change between } \\
\text { scans }\end{array}$ & $2-4$ \\
\hline $\begin{array}{l}\text { Frame-to-frame motion } \\
\text { correction }\end{array}$ & $\begin{array}{l}\text { Incorrect MBF \& MFR } \\
\text { measures }\end{array}$ & $\begin{array}{l}\text { LV position change between } \\
\text { dynamic frames }\end{array}$ & $5-9$ \\
\hline Respiratory motion correction & $\begin{array}{l}\text { Degradation of image } \\
\text { quality, artifacts }\end{array}$ & $\begin{array}{l}\text { Displacement of thorax during } \\
\text { acquisition }\end{array}$ & $10-12$ \\
\hline Intra-frame motion correction & $\begin{array}{l}\text { Degradation of image } \\
\text { quality, artifacts }\end{array}$ & $\begin{array}{l}\text { Displacement of LV during static } \\
\text { acquisition }\end{array}$ & 14,5 \\
\hline
\end{tabular}


investigators to conclude that they would not expect any degradation in image quality after motion correction. ${ }^{13}$

Another important contribution of the work of Armstrong et al is their development and validation of a quantitative figure of merit to predict the visual impact of motion on image quality which they call the "dwell fraction". ${ }^{13}$ Simplified, this metric measures the fraction of time which the heart is primarily situated in the same location within a frame to the total frame time. This figure of merit can now be used to warn the diagnostician as to the possibility of image degradation due to motion blurring and, if significant, to apply motion correction algorithms.

Perhaps the most insightful message we can take away from the article by Armstrong et al is related to their use of patient restrain to reduce patient motion. ${ }^{13}$ In their acquisition protocol all patients were positioned with both arms raised above their head, with arms supported by a Velcro strap with another wide Velcro strap wrapped around the patients' upper abdomen to minimize voluntary patient movement on the imaging couch. ${ }^{13}$ This use of restrains illustrates that those who understand the ill-effects that motion can cause to our imaging results take every precaution to first prevent and/or reduce motion as the best action to maintain image qualitative and quantitative fidelity and to then, after acquisition, quality control for detecting deleterious motions and finally correct it. Table 1 summarizes the many tools being developed and validated that have been reported to prevent, reduce and/or correct for motion-effects. These tools can be as simple as using regadenoson as the stress agent, reported to yield fewer motion artifacts compared to adenosine. ${ }^{15}$

Another important message from the article by Armstrong et al is that the important developments that they report were made in collaboration with industry. ${ }^{13}$ It is appropriate to expect and request from industry that our expensive PET scanners come equipped with hardware and software to generate optimal image fidelity, including validated motion detection and correction tools. But, it is also imperative that we work together with industry to help facilitate these developments, particularly sharing what is needed in the clinical experience. Industry also has limited resources and these collaborations will expedite the development of clinically validated tools. After all, as the often overused saying goes: "we are all in this together".

Nuclear cardiology has a large armamentarium of tools to prevent, detect and correct for motion that degrade our images and thus improve the overall quality of our clinical studies. As we have pointed out before ${ }^{16}$ these tools only have value if we used them.

\section{Disclosures}

Dr. Ernest Garcia receives royalties from the sale of the Emory Cardiac Toolbox and has equity positions with Syntermed, Inc. The terms of these arrangements have been reviewed and approved by Emory University in accordance with its conflict of interest policies. Drs. Garcia and Nye's cardiac PET motion correction research is supported by Grant R01 HL143350 (PI: EV Garcia) from the National Heart, Lung, and Blood Institute. The content is solely the responsibility of the authors and does not necessarily represent the official views of the NIH.

\section{References}

1. Klein R, DeKemp RA. Selection of PET camera and implications on the reliability and accuracy of absolute myocardial blood flow quantification. Curr Cardiol Rep 2020;22:109-21.

2. Gould KL, Pan T, Loghin C, Johnson NP, Guha A, Sdringola S. Frequent diagnostic errors in cardiac pet/ct due to misregistration of ct attenuation and emission pet images: A definitive analysis of causes, consequences, and corrections. J Nucl Med 2007;48:111221.

3. Slomka PJ, Diaz-Zamudio M, Dey D, et al. Automatic registration of misaligned CT attenuation correction maps in Rb-82 PET/CT improves detection of angiographically significant coronary artery disease. J Nucl Cardiol 2015;22:1285-95. https://doi.org/10.1007/ s12350-014-0060-9.

4. Rezaei A, Michel C, Casey ME, Nuyts J. Simultaneous reconstruction of the activity image and registration of the CT image in TOF-PET. Phys Med Biol 2016;61:1852-74.

5. Lee BC, Moody JB, Poitrasson-Rivière A, et al. Blood pool and tissue phase patient motion effects on ${ }^{82}$ rubidium PET myocardial blood flow quantification. J Nucl Cardiol 2019;26:1918-29. http s://doi.org/10.1007/s12350-018-1256-1.

6. Thompson AD, Ficaro EP, Murthy VL, Weinberg RL. Rescued diagnostic quality by motion correction of dynamic cardiac positron emission tomography (PET) perfusion images. J Nucl Cardiol 2019;26:330-2.

7. Otaki Y, Lassen ML, Manabe O, et al. Short-term repeatability of myocardial blood flow using ${ }^{82} \mathrm{Rb}$ PET/CT: The effect of arterial input function position and motion correction. J Nucl Cardiol 2019. https://doi.org/10.1007/s12350-019-01888-5.

8. Votaw JR, Packard RRS. Motion correction to enhance absolute myocardial blood flow quantitation by PET. J Nucl Cardiol 2020;27:1114-7. https://doi.org/10.1007/s12350-019-01912-8.

9. Lu Y, Liu C. Patient motion correction for dynamic cardiac PET: Current status and challenges. J Nucl Cardiol 2020;27:1999-2002. https://doi.org/10.1007/s12350-018-01513-X.

10. Kesner AL, Chung JH, Lind KE, Kwak JJ, Lynch D, Burckhardt D, et al. Validation of software gating: A practical technology for respiratory motion correction in PET. Radiology 2016;281:239-48.

11. Manwell S, Klein R, Xu T, deKemp RA. Clinical comparison of the positron emission tracking (PeTrack) algorithm with the realtime position management system for respiratory gating in cardiac positron emission tomography. Med Phys 2020;47:1713-26.

12. Feng T, Wang J, Fung G, Tsui B. Non-rigid dual respiratory and cardiac motion correction methods after, during, and before image reconstruction for 4D cardiac PET. Phys Med Biol 2016;61:15168 . 
13. Armstrong IS, Memmott MJ, Hayden C, et al. The prevalence of image degradation due to motion in rest-stress rubidium-82 imaging on a SiPM PET-CT system. J Nucl Cardiol 2021. https://d oi.org/10.1007/s12350-021-02531-y.

14. Armstrong IS, Hayden C, Memmot MJ, Arumugam P. A preliminary evaluation of a high temporal resolution data-driven motion correction algorithm for rubidium-82 on a SiPM PET-CT system. J Nucl Cardiol 2020. https://doi.org/10.1007/s12350-020-02177-2.

15. Vleeming EJ, Lazarenko SV, van der Zant FM, Pan XB, Declerck JM, Wondergem M, et al. Cardiac displacement during $13 \mathrm{~N}$
Ammonia myocardial perfusion PET/CT: Comparison between adenosine and regadenoson induced stress. J Nucl Med Technol 2018;46:114-22.

16. Garcia EV. Deep learning, another important tool for improving acquisition efficiency in SPECT MPI Imaging. J Nucl Cardiol 2020. https://doi.org/10.1007/s12350-020-02188-z.

Publisher's Note Springer Nature remains neutral with regard to jurisdictional claims in published maps and institutional affiliations. 